\title{
GUEST EDITORIAL, 14.1: The Phoenix Arises: Laser Therapy Flies Again
}

\author{
Professor Hayk Arakelyan MD PhD \\ Dean, Clinical Research Department, UNESCO Chair \\ in Life Sciences, Yerevan, Armenia
}

Dear colleagues in phototherapy and photobiomodulation, in this first Editorial for issue 14.1 of the revived Laser Therapy I would like to say first of all how humble I feel, and yet how proud to have been asked to provide the first Guest Editorial for the newly revitalized journal. I am also delighted to have been asked to become the Co-Editor of the journal for Eastern Europe and Asia. I am equally delighted to be working with illustrious colleagues, including of course the Founding Editor, and indeed interim Editor-in-Chief of the journal, Professor Toshio Ohshiro. I am ably supported in this great venture by Professor David Baxter, as the Co-Editor (Europe and Australasia), who brings a huge amount of experience to the table both academically and as an Editor-in-Chief himself of the excellent journal Physiotherapy Reviews, and Professor Ronald Wheland from the University of Arizona Medical Center, a famous name in dermatological laser circles that needs no explanations, as Co-Editor (the America's). I recommend that you log on to the journal website. The site is currently under construction, but at the very least it will let you download the Instructions for Contributors rather than having to copy them from this issue, and will also keep you right up to date with the composition of the Editorial Board and other day-by-day issues of $\mathrm{La}$ ser Therapy.

In the past five years, phototherapy has taken several major leaps forward in concept and available devices. Laser therapy systems have become more compact, but more powerful. Many are now fibreoptic-delivered, thus finishing speculation about the correct measurement of the incident power, as the fibreoptic cable forces a multimode spatial component on the laser beam, delivering a 'top hat' beam cross-section, with homogeneity which allows for accurate beam power measurement from edge to edge. Furthermore, systems capable if 1.0 W $(1000 \mathrm{~mW})$ of power are available, but with large spot sizes which allow the irradiation of larger areas than before, thus improving the irradiated volume of tissue and enhancing the therapeutic effect. The new generation of light-emitting diodes (LEDs) have added a new dimension to non-laser phototherapy, which was previously the domain of very broad waveband, xenon lamp-based systems delivering from the middle of the visible part of the spectrum through to $1200 \mathrm{~nm}$ or more in the near infrared. The latest generation of LEDs have a much improved output, both in terms of stable power and very narrow spectral outputs, plus or minus a very few nanometres, and when mounted in arrays can offer really clinical useful intensities at the recognized popular wavelengths pioneered by the helium neon $(\mathrm{HeNe})$, gallium aluminium diode (GaAlAs) and other therapeutic diode lasers.

On the science side of phototherapy, more understanding is still needed of the actual mechanisms involved, but tremendous advances have been made, based on concepts which were first published in the previous existence of this very journal. At a cellular level, the enhanced activity of pooled human neutrophils following IR LLLT was demonstrated by Dima, Shiroto and others. At a subcellular level, cytochrome $\mathrm{C}$ oxidase was identified by Tiina Karu and colleagues as the main photoacceptor for visible light in the mitochondria. At a genetic level, the IR LLLT-mediated upregulation of the genes responsible for the improved production of bone by osteoblasts and for the enhanced synthesis of adenosine triphosphate to fuel the process was first discussed by Tamura and colleagues. And of course there is much more to come in the future. It is, however, sad that the vast body of peer-reviewed literature published in Laser Therapy from Volumes 1 to 13 has not been made properly available to researchers through an internet-based abstracting service. It is one of our main goals to see Laser Therapy accepted for abstracting by Medline and Excerpta Medica (Embase) with the ultimate goal of registration with Index Medicus. Of course, this will take time and will require regular appearance of the journal, which we plan initially to be quarterly, with issues appearing in March/April, June/July, September/October and December/January. However, we have already been in touch with Medline, and we have started the registration process. Once accepted, after 2 years of the timely appearance of a high scientific content journal, although Medline will only start abstracting the journal contents from that time, all of the previous published work will then be accepted in an electronic form by PubMed so that all previous authors and their papers will be searchable in the internet.

As with previous Guest Editorials, terminology in LLLT 
has to be an important issue. It is such an important subject that I would like to reiterate some of the arguments first put forward by Ohshiro in his own Editorials, and Calderhead in his benchmark articles 'Watts a Joule' and 'Watts a Joule Revisited'. In the literature, such terms as 'low energy', 'low power', 'cool', 'cold', 'soft', and 'mid' are used to describe the systems used in laser 'biostimulation', 'bioregulation', or 'biomodulation'. None of the 'machine' terminology actually takes into account the effect of the laser therapy (as distinct to laser surgery), and it was this problem which led Ohshiro to develop his effect-based classification which is discussed elsewhere in this issue. The reaction in tissue following irradiation with laser energy can be divided into photodestructive, and photobioactive. In the first case, the level of reaction in tissue is above the threshold of irreversible damage. This damage may be thermal or nonthermal in nature. Ohshiro first referred to this as 'High reactive Level Laser Treatment', or HLLT. Note that it describes the level of the reaction between laser energy and tissue, and does not deal with the hardware used to produce that energy. If the level of reaction in tissue is nondestructive and completely reversible, Ohshiro referred to that as 'Low reactive Level Laser Therapy', or LLLT, which remains the logo of the revived Laser Therapy.

For many years, this level of reaction has been referred to in the USA and by the Federal Food and Drug Administration (FDA) as 'biostimulation': this, however, implies an increase in activity following a stimulus. Ohshiro first proposed the much better term 'bioactivation', since LLLT has been shown to work well in both hyper- and hypometabolic conditions. When one activates the accelerator in a car, the engine works harder and the car goes faster. When one activates the braking system, the car slows down and the engine ceases to work as hard. In both cases, a system is activated. However, the term 'photobiomodulation' became the accepted parlance, and I think this is also exactly right in describing the interaction between low incident levels of light and living tissue. You will all note that this has been incorporated in the subtitle of the journal, along with 'phototherapy', as we must feel free to include papers in the journal on any aspect of light-tissue reaction, irregardless of the source. However I feel very strongly, as does Dr Ohshiro, that a universal terminology will help to establish the bona fide of laser therapy, which can only further strengthen the impact of LLLT on the 'legitimate' medical and scientific community. This has been helped immensely by the recognition by the USA FDA of laser therapy through an approval early in 2002. This was what is known as a $510 \mathrm{k}$ approval, which means that the effect of the laser therapy (on carpal tunnel pain) was equivalent to an existing approved device. We in Laser Therapy would encourage manufacturers to go further, and to try for full premarketing approval based on your own data and extensive clinical trials. The FDA refer to that as a PMA, and it should absolutely be the Holy Grail of phototherapeutic system manufacturers. The first PMA issued to a phototherapy system will be another real step forward in assuring the legitimacy of phototherapy and photobiomodulation.

The nest question is probably what kind of papers are we looking for? As you will see from our Aims and Scope statement, Laser Therapy will publish papers on any aspect of photobiomodulation. The source could be a dedicated LLLT system, a defocused surgical laser, a broad band noncoherent light source or an LED system. The paper could be a double blinded, controlled clinical trial or basic science experiment, which of course are the best type of paper, but we will also consider any kind of clinical report from professionals working in all fields of medicine which includes clinicians of all specialities, veterinarians, physical and physiotherapists, dental practitioners, nurse practitioners and nursing staff, indeed any health care professional working in the field of phototherapy and photobiomodulation. Of course, parameters will remain an extremely important issue, and you must please include all of the following information; (a) The type of device, (e.g., laser therapy system, LED phototherapy system, et cetera); (b) The maker with their city, state and country; (c) The trade name of the device; (d) The wavelength or waveband (in nanometres [nm]); (e) The beam mode (pulsed, continuous wave [CW], frequency modulated). If not $\mathrm{CW}$, then please give the peak power, the average power, the frequency (in $\mathrm{Hz}$ ) and the duty cycle; (f) The output power at the tissue (in watts [W] or subunit, e.g., milliwatts [mW]; (g) The diameter of the spot (centimetres $[\mathrm{cm}]$ or the irradiated area $\left[\mathrm{cm}^{2}\right]$ ); and (h) The exposure time in seconds $[\mathrm{sec}]$ ). If you could also give the power density $\left(\mathrm{W} / \mathrm{cm}^{2}\right)$ also known as irradiance, and the energy density $\left(\mathrm{J} / \mathrm{cm}^{2}\right)$ also known as the radiant flux, that would be very helpful. Even in an uncontrolled trial, if you include all of the above parameters and a clear Materials (or Subjects) and Methods section, your paper will not be rejected, as we firmly believe that the clinical experience can help to spur the scientists to find out 'why' phototherapy has worked in that particular indication.

So, my dear colleagues, I hope that you will rally round the 'new' Laser Therapy and give us your solid support. 
For my part, I pledge to uphold the high quality of the journal, and with my contacts in Eastern Europe, I am sure that I will be able to bring some of the excellent science being carried out in these countries into English in our new journal, many of which studies have hitherto not been published in the English Language, and thus remain unknown in the West. Together with this resuscitated Laser Therapy, there is a strong momentum to re-establish the International Laser Therapy Association (ILTA), and in fact this journal has been accepted as their official journal, just as it was in 1988 when Laser Therapy first appeared. Let's move forward together, with both a society and a journal absolutely dedicated to all aspects of the excellent science and clinical practice of phototherapy and photobiomodulation!

Yerevan, Armenia. March 2005 